\title{
Analysis of a Novel Resistive Film Absorber for Suppression of Electromagnetic Radiation in System-in-Packages
}

\author{
Shuyun Huo $\mathbb{D},{ }^{1}$ Yan Li $\mathbb{D}^{2},{ }^{2}$ Xiaoyong Lei $\mathbb{D}^{1},{ }^{1}$ Zhe Sun $\left(\mathbb{D},{ }^{1}\right.$ Heyuan Yu $\mathbb{D},{ }^{1}$ Bingheng Li $\left(\mathbb{D},{ }^{3}\right.$

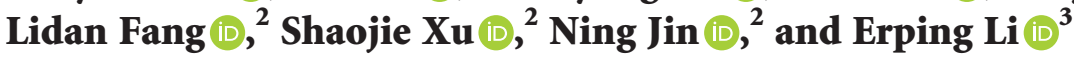 \\ ${ }^{1}$ The School of Electronics and Information Engineering, Hebei University of Technology, Tianjin 300400, China \\ ${ }^{2}$ Key Laboratory of Electromagnetic Wave Information Technology and Metrology of Zhejiang Province, \\ College of Information Engineering, China Jiliang University, Hangzhou 310018, China \\ ${ }^{3}$ The Key Laboratory of Advanced Micro-Nano Electronic Devices and Smart Systems and Applications, \\ College of Information Science and Electronic Engineering, Zhejiang University, Hangzhou 310027, China \\ Correspondence should be addressed to Yan Li; liyan@cjlu.edu.cn
}

Received 6 November 2021; Revised 5 January 2022; Accepted 20 January 2022; Published 7 March 2022

Academic Editor: Atsushi Mase

Copyright (C) 2022 Shuyun Huo et al. This is an open access article distributed under the Creative Commons Attribution License, which permits unrestricted use, distribution, and reproduction in any medium, provided the original work is properly cited.

\begin{abstract}
This paper presents a new method to suppress the electromagnetic radiation between the heatsink and packaging substrate in the system-in-package by using a resistance film absorber. The proposed absorber is designed with the indium tin oxide sputtered on both sides of the glass substrate, and the top layer adopts the combination of Jerusalem cross-shaped, ring-shaped, and L-shaped resistive film to expand the bandwidth. The unit size of the absorber is $0.14 \lambda_{L} \times 0.14 \lambda_{L}$ and the thickness is $0.049 \lambda_{L}$. It has an absorptivity of more than $90 \%$ in the frequency range of $21 \mathrm{GHz}$ to $55 \mathrm{GHz}$ with polarization insensitivity, and angular stability. Moreover, the radiated electric field from the chip package at $3 \mathrm{~m}$ is significantly reduced when employing the proposed absorber, and the maximum suppression of the electric field reaches $18 \mathrm{~dB}$. Finally, the measurement results are carried out to verify the simulation results. Both simulation and experiment results demonstrate that the proposed absorber has excellent radiation suppression, which can be properly applied to electromagnetic interference suppression of the printed circuit board.
\end{abstract}

\section{Introduction}

With the continuous improvement of the operating frequency and integration density of RF chips, electromagnetic interference and heat dissipation gradually become a great challenge for electronic devices [1]. The heatsink is usually mounted on packages to dissipate heat from ICs to free space [2]. As the package lid and heatsink are close to the surface of ICs, part of the noise will be coupled to the package lid and heatsink through the electric field. Since a resonant cavity is formed between the ground plane and heatsink, the electromagnetic wave is reflected back and forth between the two metal planes, resulting in a substantial enhancement of electromagnetic radiation [3]. There are gaps between the package lid/heatsink and the chip package substrate to provide sufficient space for the cables and components in the circuit system, which makes radiation leak out through these gaps, and results in radiation exceedance [4].

The grounding post is used to solve the problems caused by the package lid and heatsink. Due to the parasitic effect of grounding posts, this method only works well at low frequency and has no obvious suppression at high frequency, which is also constrained by high-density layout [5]. The radiation-suppression methods also include the use of absorbing material, which is coated on the surface of signal alignments or placed around the package substrate [6]. However, the absorbing properties are affected by temperature, and poor thermal conductivity of absorbing material results in ineffective heat exchange with the external environment [7]. The application of electromagnetic band-gap (EBG) structures for EMI suppression has also received high attention, which is a breakthrough technique to achieving broadband and high isolation noise suppression [8]. The 
EBG structure maintains the integrity of the power supply and ground planes, but the size is too large when applied to the high-density-layout space between the heatsink and the package substrate [9].

Metamaterial absorbers provide solutions to the aforementioned problems. Compared with traditional absorbing technology, metamaterial absorbers have the advantages of simple structure, lightweight, and high absorptivity $[10,11]$, which become an important research direction of electromagnetic absorbing, but the traditional absorbers based on the resonant of metal structures have a less-stable equivalent impedance, and at deviations from the resonant frequency, the surface input impedance of the absorber is poorly matched to the impedance of free space, and the effective absorption bandwidth is too narrow to be suitable for engineering applications [12]. The high surface resistance film pattern not only has electromagnetic filtering characteristics but also has a more stable surface impedance, the absorption characteristics of the metamaterial absorber can be further improved by rational designing the resistive film structure to achieve effective adjustment of the resonance characteristics [13].

In this paper, a miniaturized resistive film absorber is presented. The top layer uses a Jerusalem cross-shaped, ringshaped, and L-shaped structure to enhance the coupling, resulting in the absorber having an absorptivity of more than $90 \%$ in the frequency range of $21 \mathrm{GHz}$ to $55 \mathrm{GHz}$. The electric field and surface current distribution are analyzed, and the operating mechanism is explored by combining the equivalent medium theory and equivalent circuit analysis, and the absorption performance is verified experimentally. The proposed absorber has a high electromagnetic absorptivity, which can significantly reduce the electromagnetic radiation from the IC packages system.

\section{Radiation Analysis of IC Packages}

2.1. IC Packages Structure. In order to effectively suppress the radiation emission caused by IC packages and heatsinks, it is necessary to analyze the radiation mechanism. The practical flip-chip package consists of multiple power, ground planes, and high-density layout, so the packaging structure is very complex and difficult to analyze accurately in theory. In this paper, a part of the package model is utilized to analyze the radiated emission effects of the heatsink.

As shown in Figure 1, the simplified package system model consists of flip-chip, heatsink, and PCB. To reduce the thermal resistance between the heatsink and the chip, a highly thermal conductive pad is used between the two components to make heat dissipation in the package easier. The size of the thermal conductive pad is $9.7 \mathrm{~mm} \times 11 \mathrm{~mm} \times 0.95 \mathrm{~mm}$, and the size of the PCB is $10 \mathrm{~cm} \times 10 \mathrm{~cm} \times 1.6 \mathrm{~mm}$. The horizontal dimension of heatsink is $3.3 \mathrm{~cm} \times 3.3 \mathrm{~cm}$, the bottom metal thickness is $3 \mathrm{~mm}$, and the space between the top of the PCB and the bottom of the heatsink is $1.6 \mathrm{~mm}$. The dimension of fins are as follows: the height is $7.5 \mathrm{~mm}$, the thickness is $3 \mathrm{~mm}$, the width is $1 \mathrm{~mm}$, the spacing is $1.6 \mathrm{~mm}$, and the fins number is
121. In this paper, the simulation and experiment are implemented when set aggregated port 1 and port 2 for both arms of the LC tank inside the DIE, as shown in Figure 2, and we set the excitation current to $200 \mathrm{~mA}$ in CST.

2.2. Radiation of Heatsink. For the system consisting of IC package, $\mathrm{PCB}$, and heatsink, the IC inside the chip is the noise source. The heatsink and PCB on the power/ground plane constitute an effective radiation antenna. The noise source coupled to free space by radiation field, resulting in EMI radiation exceeding the standard. The radiation from the heatsink can be regarded as two parts: the cavity radiation component is dominant in short heatsink fins with large cross-sections, and the monopole component is dominant when the heatsink fins are relatively long [14].

We simulated the $3 \mathrm{~m}$ electric field with and without a heatsink, respectively. As shown in Figure 3, the effect of with or without heatsink fins on the far-field results has less impact, which means the cavity radiation component plays a dominant role in the radiation. In the follow-up study, the heatsink is modeled as a metal block to save simulation resources.

\section{Structural Design and Numerical Simulation}

3.1. The Design Theory. Traditionally, according to the law of energy conservation, when the electromagnetic wave is incident on the surface of the absorber structure, the energy is divided into three parts, including transmitted energy $T(\omega)$, reflected energy $R(\omega)$, and absorbed energy $A(\omega)$ [2]. The absorbed energy can be described as

$$
A(\omega)=1-R(\omega)-T(\omega)
$$

where $R(\omega)=\left|S_{11}\right|^{2}$ and $T(\omega)=\left|S_{21}\right|^{2}$. The backboard of the absorber is completely covered by metal, making incident wave unable to transmit, the transmission coefficient $S_{21}=0$, confirming that the absorbed energy can be expressed as

$$
A(\omega)=1-R(\omega)=1-\left|S_{11}\right|^{2} .
$$

The reflection coefficient $S_{11}$ determines the absorption performance of the structure. When $S_{11}=0$, the model can achieve the complete absorption in the corresponding band.

3.2. Design of the Proposed Absorber Structure. The absorber designed in this paper is composed of a resistive film periodic array on the top, a dielectric substrate in the middle, and a grounding layer at the bottom. The unit cell structure is shown in Figure 4, the top layer is the indium tin oxide (ITO) resonant structure sputtered on the glass substrate, and the dielectric constant of substrate is 6 , the bottom layer is a continuous ITO grounding layer sputtered onto the other surface of the glass substrate, with a square resistance value of $5 \Omega / \mathrm{sq}$, which provides a zero transmission $\left(S_{21} \approx 0\right)$, the whole absorber forms an ITO-glass-ITO Sandwich structure. To achieve broadband absorption, the design of the absorber is mainly focused on the top patch, the ITO on the top layer has a resistance of $250 \Omega / \mathrm{sq}$, the pattern is 


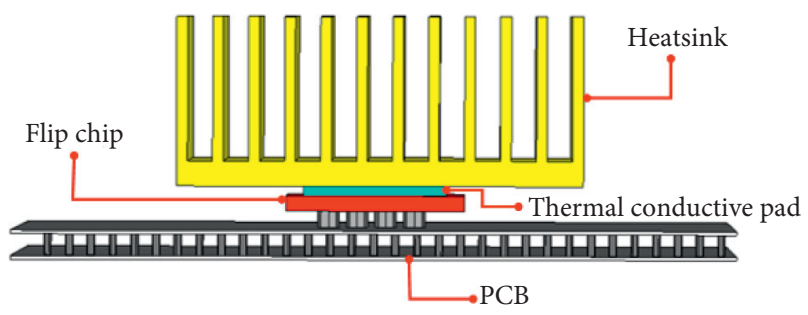

(a)

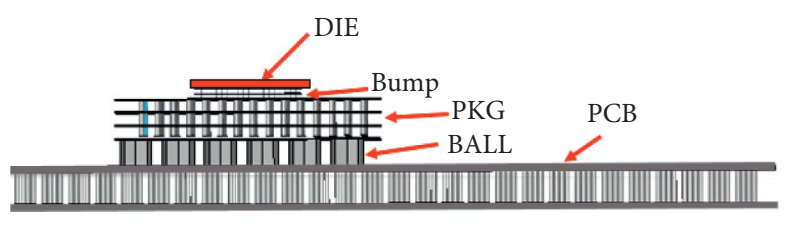

(b)

Figure 1: IC package: (a) simplified packaging model and (b) part of the packaging model without heatsink.

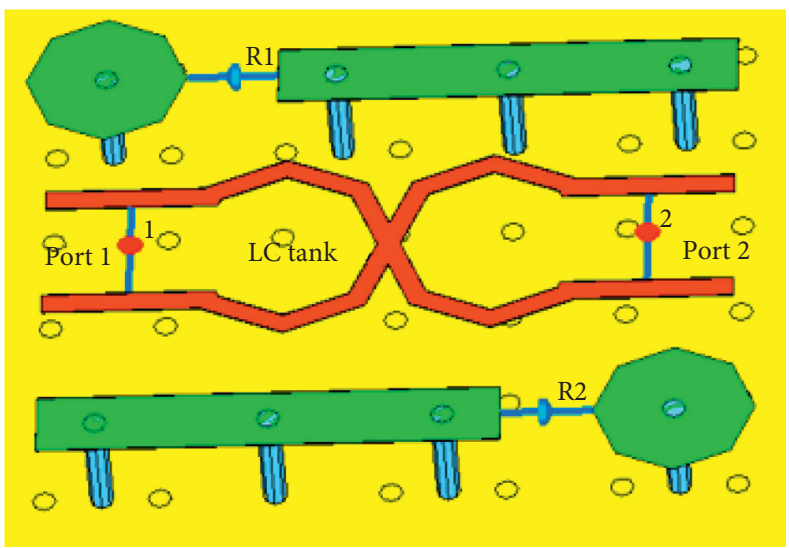

FIgURE 2: Excitation of packaging model.

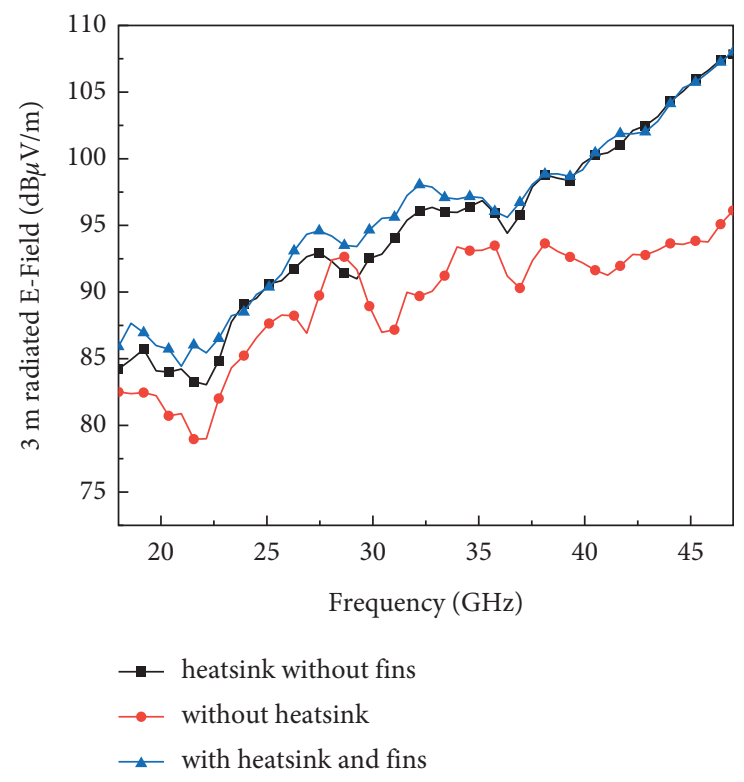

FIGURE 3: $3 \mathrm{~m}$ E-field with or without heatsink and fins.

designed as a Jerusalem cross shape combined with a square ring, and four L-shaped structures are added at each corner to enhance the coupling between adjacent cells, and the values of the geometric parameters of each part are shown in Table 1 .
3.3. Numerical Simulation. The proposed structure is modeled and simulated by a frequency domain solver based on CST. We set the unit cell boundary in the $x$ - $y$ plane and excitation ports in the wave propagation direction $z$ to simulate uniform plane wave incident on the designed structure, and the boundary along the $z$-direction is set to open (add space). As the preparation environment and 


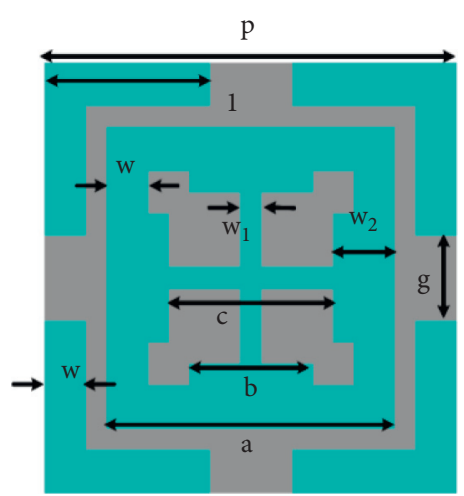

(a)

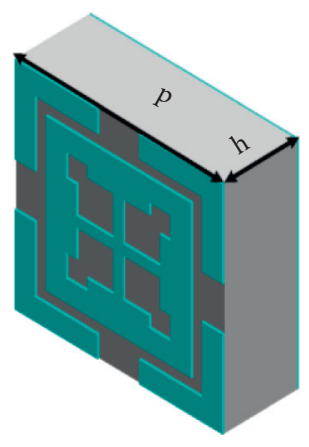

(b)

FIgURE 4: Structural diagram of the proposed absorber: (a) front view and (b) side view.

TABLE 1: Geometric parameters of the proposed structure.

\begin{tabular}{lccc}
\hline Parameters & Value $(\mathrm{mm})$ & Parameters & Value $(\mathrm{mm})$ \\
\hline P & 2.0 & $\mathrm{w}$ & 0.2 \\
L & 0.8 & w1 & 0.1 \\
A & 1.4 & w2 & 0.3 \\
B & 0.4 & h & 0.7 \\
C & 0.8 & g & 0.4 \\
\hline
\end{tabular}

chemical ratios of ITO will directly affect its dielectric coefficient, permeability, and loss angle tangent, the ITO material is set as the impedance boundary, the resistance is set as the magnitude of the surface resistance value, and the reactance is set as 0 .

The obtained copolarized and cross-polarized reflection coefficient $S_{11}$ (left column) are shown in Figure 5. We found that the cross-polarized reflection coefficient is less than $-80 \mathrm{~dB}$ and the copolarized reflection coefficient is less than $-10 \mathrm{~dB}$ in the range from $21.0 \mathrm{GHz}$ to $55.0 \mathrm{GHz}$. Therefore, when a plane wave with horizontal (or vertical) polarization is incident into the absorber structure, less of the incident wave undergoes polarization conversion, whereas most of the incident wave is absorbed. We calculate the absorptivity by equation (2), as shown in Figure 5 (right column), ultrabroadband absorption is achieved in the frequency from $21.0 \mathrm{GHz}$ to $55.0 \mathrm{GHz}$, with an absorptivity greater than $90 \%$, and a relative absorption bandwidth reaches $89.5 \%$.

To verify the stability of the polarization angle of the resistive film absorber, the polarization angle $\varphi$ is changed to observe the absorptivity under different polarization modes, as shown in Figure 6. The absorption distribution under different polarizations is the same when the polarization angle is changed, which is determined by the symmetry of the proposed structure.

The angular stability of the absorbing structure is required because of the complex variety of radiation sources, and the miniaturized design is more conducive to the angular stability of the absorber. The polarization direction and the incident angle are illustrated in Figure 7(a). Figures 7(b) and 7 (c) show the effect of the absorptivity of TM and TE

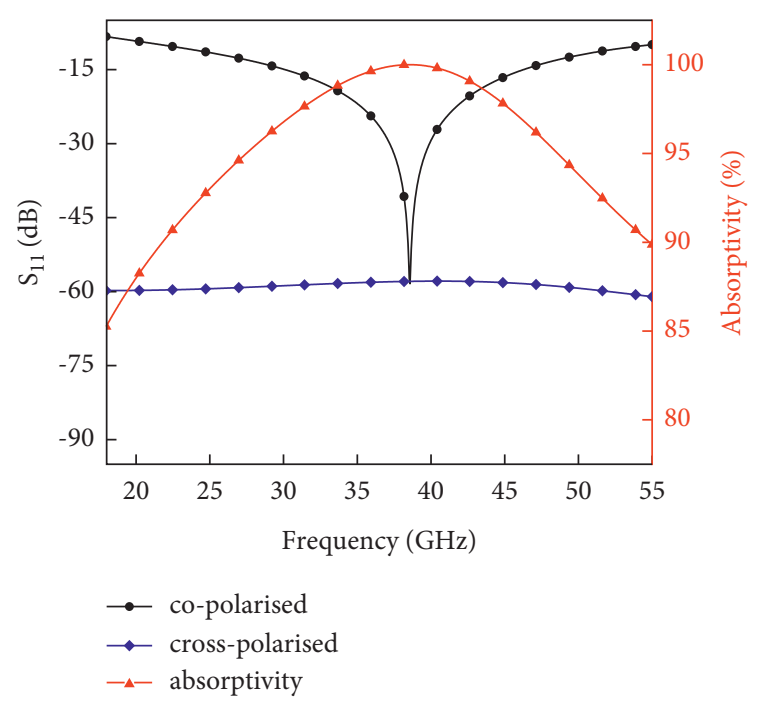

FIgURE 5: Simulated copolarized and cross-polarized S11 (left column) and absorptivity (right column).

polarizations when the incidence angle $\theta$ is increased from $0^{\circ}$ to $60^{\circ}$. For the TE polarization, when the incidence angle increases from $0^{\circ}$ to $45^{\circ}$, the absorptivity remains above $90 \%$ between $21 \mathrm{GHz}$ and $55 \mathrm{GHz}$. When the incidence angle $\theta$ is $60^{\circ}$, the absorptivity decreases but remains above $70 \%$ between the aforementioned frequency band. When the incidence angle is changed in TM polarization, and the absorptivity remains above $85 \%$ between $15 \mathrm{GHz}$ and $55 \mathrm{GHz}$ when the incidence angle is $60^{\circ}$. Angular stability of TM polarization is better than TE polarization. 


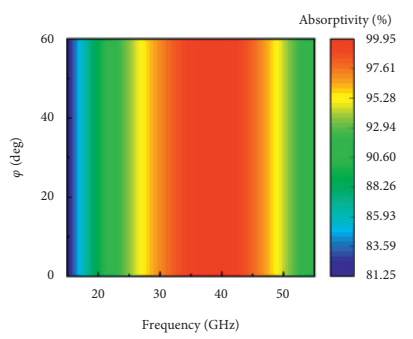

(a)

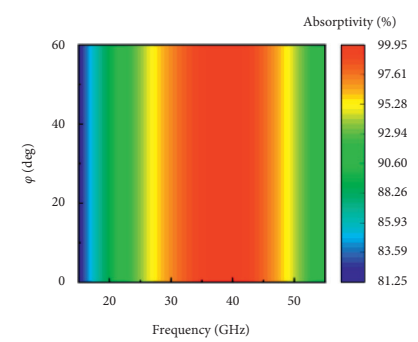

(b)

FIgURE 6: Absorptivity in TE and TM polarizations at different polarizing angle: (a) TE polarization and (b) TM polarization.

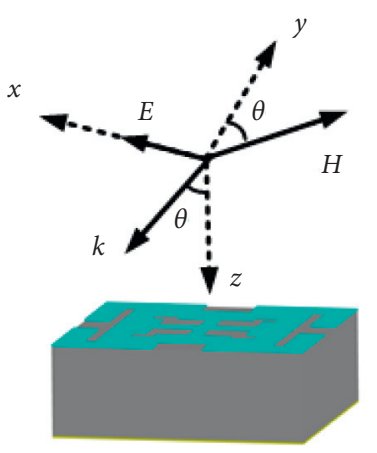

TE

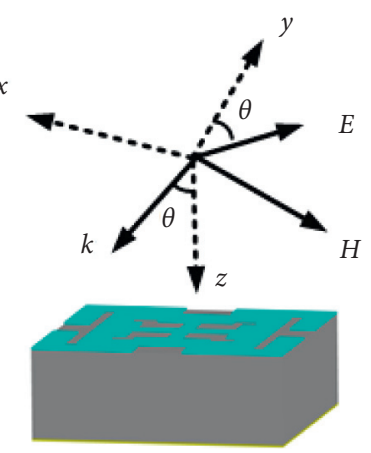

TM

(a)

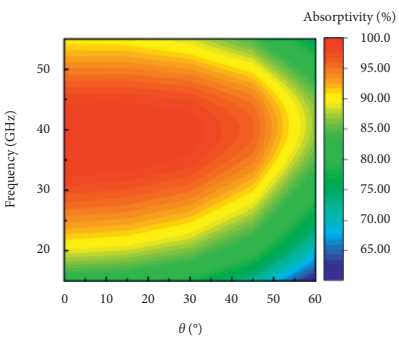

(b)

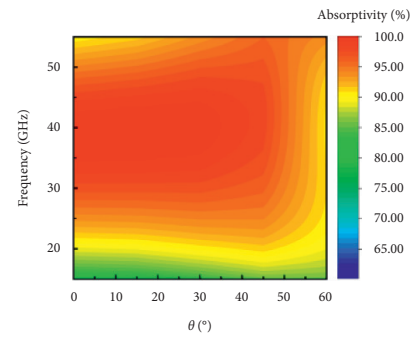

(c)

Figure 7: (a) Schematic view of the proposed absorber at oblique incidences, (b) absorptivity of TE polarization at different incident angle, and (c) absorptivity of TM polarization at different incident angle.

\section{Analysis of the Absorption Mechanism}

Two conditions must be met to achieve the perfect absorption. The first condition is the maximum amount of incident wave into the absorber to reduce the reflection of incident wave, and match the impedance between the absorber and the free space in the widest frequency range. The second condition is that the absorber can produce effective loss, converting electromagnetic wave energy into heat or other forms of energy.

4.1. Impedance Matching. To verify that the designed structure achieves impedance matching, the equivalent medium theory is used to equate the absorber to a homogeneous medium, and its corresponding equivalent impedance is calculated by the $S$ parameter inversion method.

When a uniform plane wave is incident perpendicular to an infinite medium plate of thickness $d$ [15], the reflection and transmission coefficients are

$$
\begin{aligned}
& \frac{1}{t}=\left[\cos (\mathrm{nkd})-\frac{j}{2}\left(z-\frac{1}{z}\right) \sin (\mathrm{nkd})\right] e^{\mathrm{jkd}} . \\
& \frac{r}{t^{\prime}}=-\frac{1}{2} j\left(z-\frac{1}{z}\right) \sin (\mathrm{nkd}),
\end{aligned}
$$

where $t^{\prime}=t \exp (j k d)$ is the transmission coefficient and $r$ is the reflection coefficient, and $k$ denotes the free space propagation constant. Equations (3) and (4) can be organized as

$$
z= \pm \sqrt{\frac{\left(1+r^{2}\right)-t^{\prime 2}}{\left(1-r^{2}\right)-t^{\prime 2}}}
$$

Equation (5) is the relative impedance of the proposed structure, as is shown in Figure 8, which is obtained by substituting the scattering coefficient obtained from the simulation into equation (5). In the frequency range of $21 \mathrm{GHz}$ to $55 \mathrm{GHz}$, the real and imaginary parts of input impedance are approximately 1 and 0 respectively, which indicates that the designed structure achieves impedance matching with the free space.

4.2. Mechanism of Absorption. To study the absorption mechanism, the distribution of electric field and surface current is investigated at the frequency of the absorption peak. Figure 9(a) and 9(b) shows the surface current distribution of the top and bottom layers at $39 \mathrm{GHz}$, respectively. The electromagnetic wave generates a strong charge buildup on the surface of the ITO film; the induced surface current is mainly concentrated at the middle of the square ring and the L-shaped arms. The ITO film interacts with the bottom layer to form an antiparallel current, while the displacement current is generated in the substrate. The induced current and displacement current eventually form circulating current loops and generate magnetic resonance to manipulate the relative permeability. 


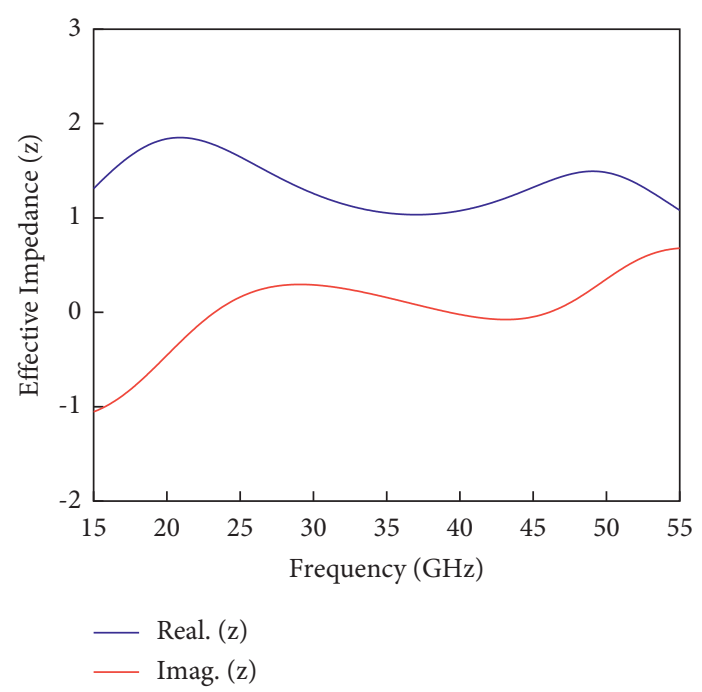

FIGURE 8: The relative impedance of the proposed structure.

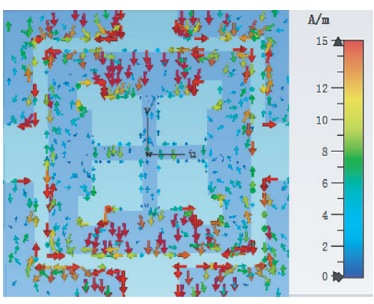

(a)

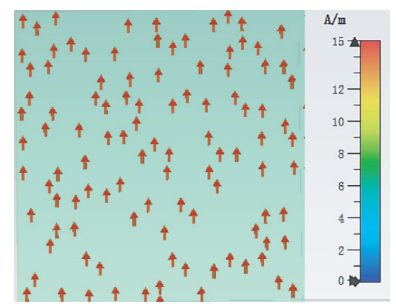

(b)

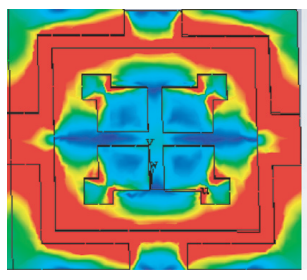

(c)
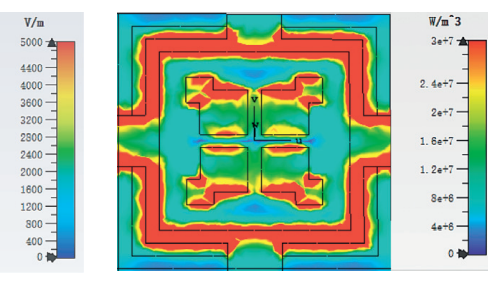

(d)

Figure 9: (a) The top surface current distribution; (b) the current distribution on the ground; (c) electric field distribution; and (d) energy loss distribution of the absorber.

Figure 9(c) shows the $z$-component of $E$-field distributions at the resonant frequency, the electric field is bound between the L-shaped and ring-shaped, where a strong capacitive coupling exists and the resulting electric resonance can effectively control the dielectric constant.

In Figure 9(d), the energy loss distribution for the proposed absorber surface is similar to the electric field distribution, which means that the polarization of ITO and glass substrate occurs under the action of the induced electric field, and the electric dipole in the substrate will be arranged in the same direction as the electric field, the electric dipole in the rotation and friction of the polarization process will convert microwave energy to heat.

Electromagnetic loss capability can be characterized by the loss tangent $\tan \delta$; the larger the loss tangent, the better the absorption performance. The electric loss tangent is $\tan \delta e=\varepsilon^{\prime \prime} / \varepsilon^{\prime}$, the magnetic loss tangent is $\tan \delta m=\mu^{\prime \prime} / \mu^{\prime}$, where $\varepsilon^{\prime}$ represents the ability to store electric charge, and the $\varepsilon^{\prime \prime}$ represents the ability to lose energy. $\mu^{\prime}$ denotes the magnetic energy density stored in the absorber, and $\mu^{\prime \prime}$ denotes the magnetic loss capability. Therefore, increasing the imaginary part of the equivalent dielectric constant and permeability or decreasing their real part will increase the loss factor. The equivalent dielectric constant $\varepsilon_{\text {eff }}$ and permeability $\mu_{\text {eff }}$ are calculated using equations (6) and (7), respectively [16].

$$
\begin{aligned}
& \varepsilon_{\text {eff }}=1+\frac{2 j}{k_{0} h}\left(\frac{1-r}{1+r}\right), \\
& \mu_{\text {eff }}=1+\frac{2 j}{k_{0} h}\left(\frac{1+r}{1-r}\right),
\end{aligned}
$$

where $h$ denotes the thickness of the substrate and $k_{0}$ denotes the free space wave number. The real part of the equivalent dielectric constant and permeability got by equations (6) and (7) are shown in Figure 10(a). We can see that the real part of $\varepsilon_{\text {eff }}$ and $\mu_{\text {eff }}$ are closer in the range of the absorption band, which proves the absorber structure and the free space achieve impedance matching. The imaginary part of $\varepsilon_{e f f}$ and $\mu_{\text {eff }}$ in Figure 10(b) shows that the loss is larger in the range of the absorption band.

For conventional single-structure metamaterial absorbers, the strong absorption mainly results from strong electrical and magnetic resonance, which induces ohmic loss and dielectric loss [13]. To explore the cause of energy loss, the absorptivity of the dielectric substrate with and without loss, and the absorptivity of the top layer loaded with resistive film or PEC is compared. As shown in Figure 11(a), the trend of the absorption curves is the same when the dielectric substrate is with and without loss, which means that the designed resistive film absorber has a small dielectric loss. Figure 11(b) compares the absorptivity when the top 


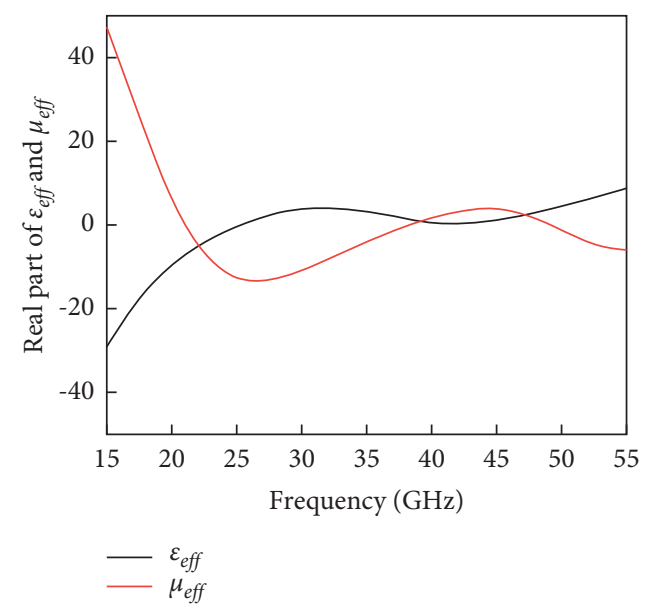

(a)

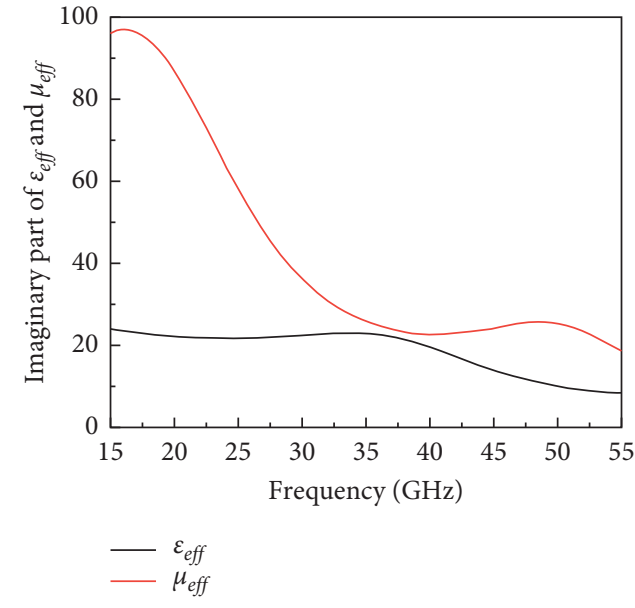

(b)

FIgURE 10: Equivalent constitutive parameters: (a) real part of $\varepsilon_{\mathrm{eff}}$ and $\mu_{\mathrm{eff}}$ and (b) imaginary part of $\varepsilon_{\mathrm{eff}}$ and $\mu_{\mathrm{eff}}$.

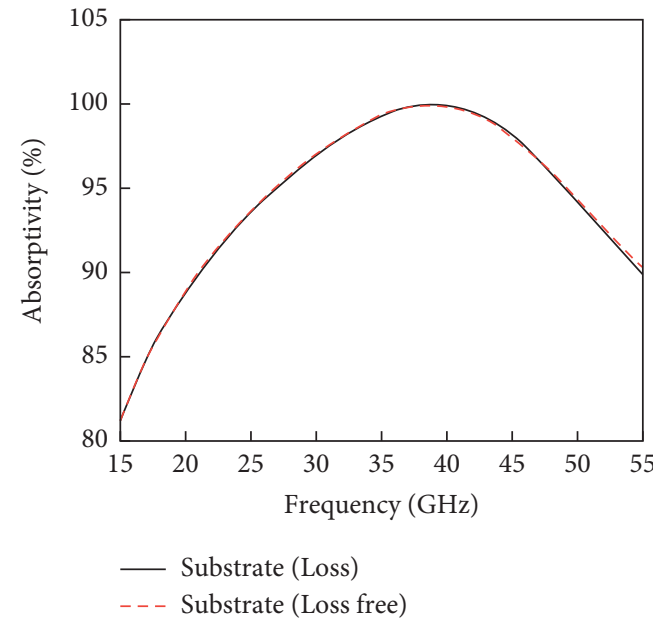

(a)

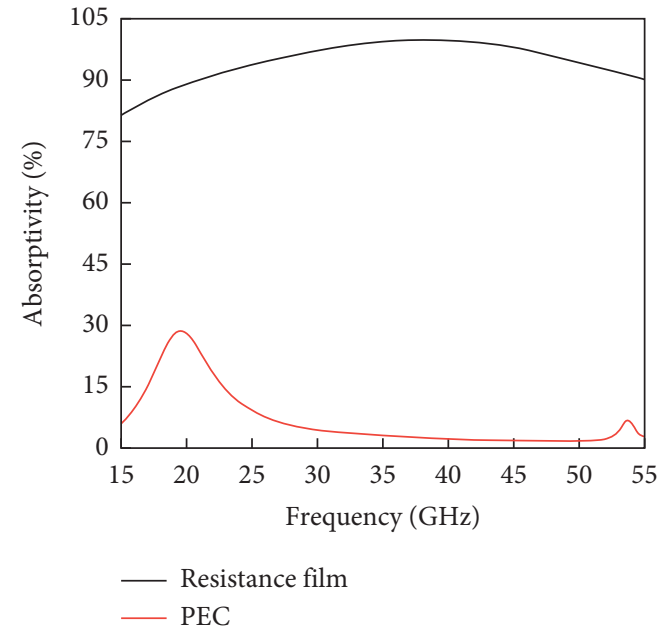

(b)

FIgURE 11: (a) Absorptivity with or without loss of the substrate and (b) absorptivity under different top layer material.

layer is ITO film or PEC. When the substrate with PEC, the absorptivity in the range of $15 \mathrm{GHz}$ to $55 \mathrm{GHz}$ is less than $30 \%$, whereas the ITO film shows strongly absorbing, so the proposed structure achieves perfect absorption mainly through ohmic loss.

4.3. Equivalent Circuit Analysis. The transmission characteristics of the resistive film absorber are described by the impedance of the equivalent circuit model, as shown in Figure 12. When the electromagnetic wave is incident vertically, the input impedance of the grounded dielectric plate is equivalent to an inductance, and the expression is

$$
\begin{aligned}
Z_{h} & =j \frac{Z_{0}}{\sqrt{\varepsilon_{r}^{\prime}+j \varepsilon_{r}^{\prime \prime}}} \tan \left(k_{0} \sqrt{\varepsilon_{r}^{\prime}+j \varepsilon_{r}^{\prime \prime}} h\right), \\
\varepsilon_{r} & =\varepsilon_{\rho}^{\prime}+\varepsilon_{r}^{\prime \prime},
\end{aligned}
$$

where $Z_{0}$ is the free-space characteristic impedance, $\varepsilon_{r}$ is the dielectric constant of the substrate, $k_{0}$ is the free-space propagation constant, and $h$ is the thickness of the dielectric substrate. The real and imaginary parts of the input impedance are expressed as

$$
\begin{aligned}
\operatorname{Re}\left(Z_{h}\right)= & \frac{Z_{0}}{\sqrt{\varepsilon_{r}^{\prime}}}\left[\frac{\varepsilon_{r}^{\prime \prime}}{2 \varepsilon_{r}^{\prime}} \operatorname{tg}\left(k_{0} h \sqrt{\varepsilon_{r}^{\prime}}\right)\right. \\
& -\left(k_{0} h \frac{\varepsilon_{r}^{\prime \prime}}{2 \sqrt{\varepsilon_{r}^{\prime}}}\right)\left(1+\operatorname{tg}^{2}\left(k_{0} h \sqrt{\varepsilon_{r}^{\prime}}\right)\right), \\
\operatorname{Im}\left(Z_{h}\right)= & \frac{Z_{0}}{\sqrt{\varepsilon_{r}^{\prime}}}\left[\operatorname{tg}\left(k_{0} h \sqrt{\varepsilon_{r}^{\prime}}\right)\right], \\
Z_{t}= & \frac{1-\omega^{2} \mathrm{LC}}{j \omega C} .
\end{aligned}
$$




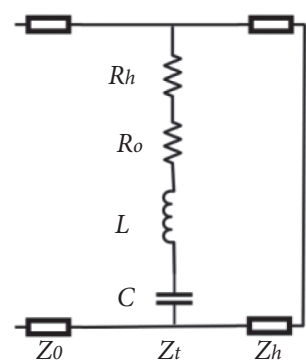

FIgURE 12: Equivalent circuit model.

If the substrate is thick enough $(h>0.3 p)$, for a lossy dielectric substrate, the loaded capacitance reads

$$
C^{\prime}=C\left[\frac{\left(\varepsilon_{r}^{\prime}+1\right)}{2}+j \frac{\varepsilon_{r}^{\prime \prime}}{2}\right] .
$$

Since the imaginary part of the capacitance in the lossy substrate brings a resistive component, this capacitance is divided into two parts: a resistive $R_{h}$ and a lossless capacitance.

$$
R_{h} \simeq \frac{-\varepsilon_{r}^{\prime \prime}}{\omega C\left(\varepsilon_{r}^{\prime}+1\right)} .
$$

Due to the presence of ohmic loss, a series resistor Ro is added to the aforementioned circuit, it can be described as

$$
R_{o}=\frac{S_{\text {top }}}{S_{\text {cell }}} R_{s}
$$

Figure 11(a) shows that the absorptivity of the structure is almost unchanged for the case of a lossless substrate, $\operatorname{Re}\left(Z_{h}\right) \approx 0$, then the real part of the absorber input impedance can be expressed as

$$
\operatorname{Re}\left\{Z_{\text {in }}\right\}=\frac{Z_{0}^{2} / \varepsilon_{r}^{\prime}\left[\operatorname{tg}^{2}\left(k_{0} h \sqrt{\varepsilon_{r}^{\prime}}\right)\right]}{S_{\text {top }} / S_{\text {cell }} R_{s}+-2 \varepsilon_{n}^{\prime \prime} / \omega_{0} C\left(\varepsilon_{r}^{\prime}+1\right)^{2}} .
$$

where $\omega_{0}$ is the first resonant frequency, equation (13) shows that the parameters affecting the input impedance of the absorber include the thickness of the substrate, the dielectric constant, capacitance $C$, and ITO square resistance $R_{S}$. The influence of these parameters on the absorption mechanism will be discussed in detail below.

4.3.1. Substrate Thickness. The reflection coefficient of the structure at three different substrate thicknesses and the input impedance of the absorber is given in Figure 13. We can see that the input impedance corresponding to the resonant frequency is equal to the free-space impedance $(377 \Omega$ ), and as the thickness increases, the resonance moves to a lower frequency due to the increase in substrate inductance. And an almost perfect match is achieved when the

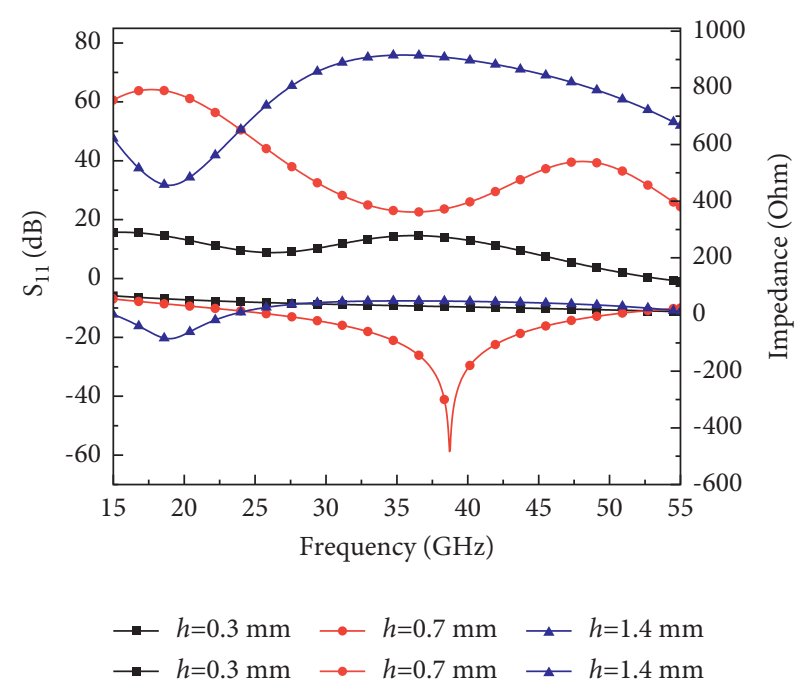

FIGURE 13: Reflection coefficient (left column) and input impedance (right column) at different substrate thicknesses.

substrate thickness is $0.7 \mathrm{~mm}$, while lower and thicker substrates lead to a mismatch.

4.3.2. Dielectric Constant. In Figure 14, the reflection coefficient and input impedance of the absorber structure are reported at three different dielectric constants. With the increase of dielectric constant, the resonant frequency moved to a lower frequency, so the absorption frequency can be adjusted by changing the dielectric constant of the substrate.

4.3.3. Unit Cell Shape. The design of the top layer is a key task in achieving an ultra-wideband metamaterial absorber. Keeping the substrate thickness fixed, the patch components require higher dielectric loss than cross-array components [17]. The reason for this behavior is that the higher field strength is coupled out on the cross-array or square ring. Based on the aforementioned conclusions, we take the cross-shape as base structure, and the loss tangent angle of the substrate is set to 0 . As shown in Figure 15, the highest absorptivity is below $80 \%$ when only the crossshaped element is analyzed. To further enhance the coupling of the top layer, we added a ring-shaped outside the cross-shaped, the absorptivity increases significantly. Moreover, to enhance the coupling between adjacent unit cells, L-shaped structures are designed at four corners, the electric field coupling between the L-shaped and ringshaped structures results in a significant improvement in absorption.

4.3.4. ITO Square Resistance. Our analysis of the working mechanism in section 4.2 shows that the energy loss is mainly concentrated on the ITO film. To enhance the absorption characteristics of the structure, it is necessary 


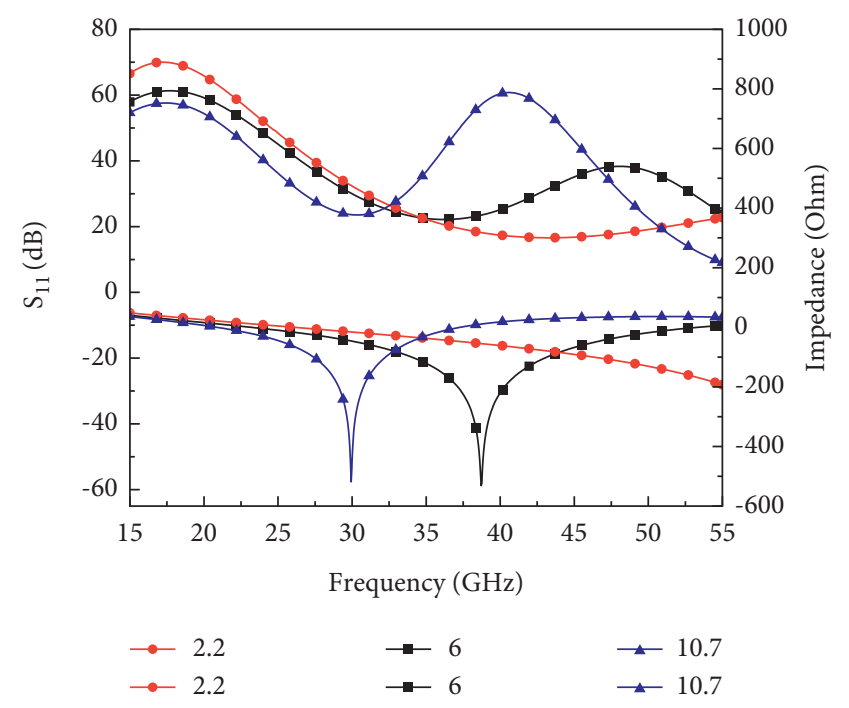

FIGURE 14: Reflection coefficient (left column) and input impedance (right column) at different substrate dielectric constant.

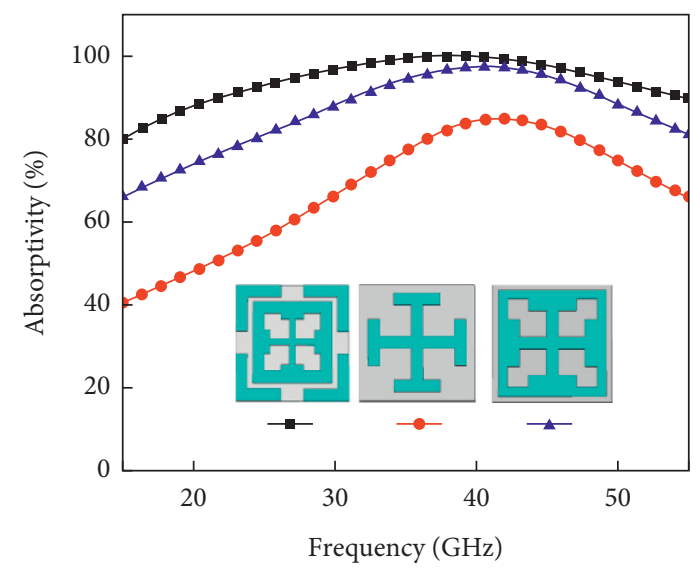

FIGURE 15: Absorptivity with different component combinations.

to design a reasonable resistance value. The absorption response of the proposed structure to different ITO surface resistance values is studied, as shown in Figure 16, when the top square resistance is $250 \Omega /$ sq, the corresponding curve has a wider working band, where the absorber achieves the best absorption effect.

4.4. Multireflection Interference Theory. We introduce the interference theory to analyze the broadband absorption mechanism. Figure 17 shows the multiple interference theory model for vertical and oblique incidence, dividing the free space and the absorber into two areas and two layers. We assume that $r_{11}$ is the reflection coefficient of pattern layer from area 1 to area $2, r_{21}$ is the transmission coefficient of pattern layer from area 1 to area $2, r_{12}$ is the transmission coefficient of pattern layer from area 2 to area 1 , and $r_{22}$ is the reflection coefficient of pattern layer from area 2 to area 2 , which are described as

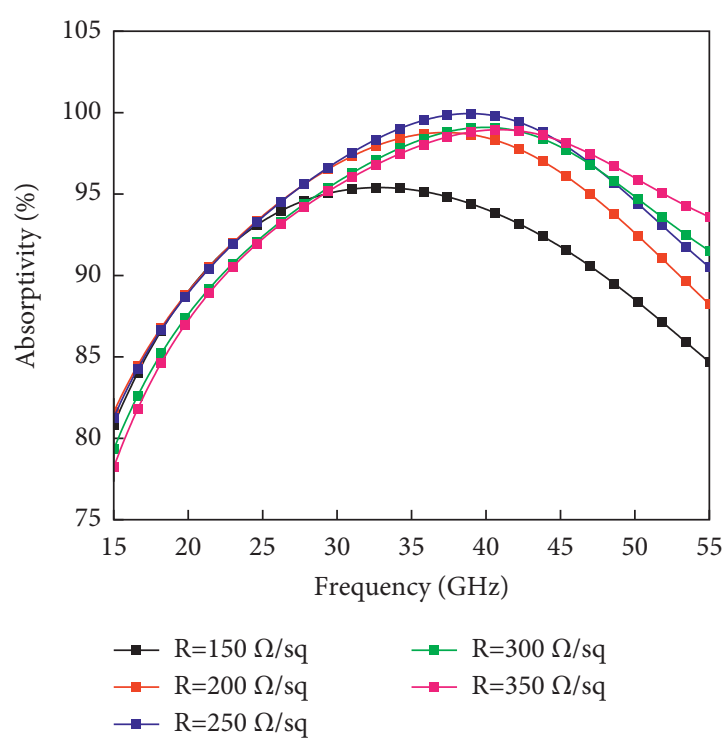

FIgURE 16: The absorptivity with different square resistance.

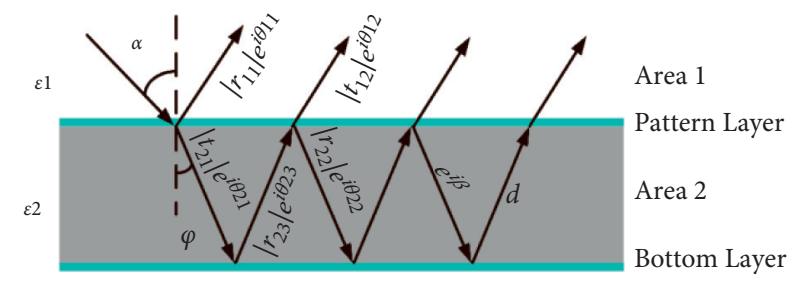

FIgURE 17: Theoretical model of multiple reflection with vertical and oblique incidence.

$$
\begin{aligned}
& r_{11}=\left|r_{11}\right| e^{j \theta_{11},} \\
& r_{21}=\left|r_{21}\right| e^{j \theta_{21},} \\
& r_{12}=\left|r_{12}\right| e^{j \theta_{12}}, \\
& r_{22}=\left|r_{22}\right| e^{j \theta_{22}} .
\end{aligned}
$$

The total reflected wave of pattern layer returning from area 1 to area 1 due to the presence of the ground plane is

$$
\begin{aligned}
r= & r_{11}+r_{21} e^{i \beta} r_{23} e^{i \beta} t_{12}+t_{21} e^{i \beta} r_{23} e^{i \beta}\left(r_{22} e^{i \beta} r_{23} e^{i \beta}\right) t_{12} \\
& +t_{21} e^{i \beta} r_{23} e^{i \beta}\left(r_{22} e^{i \beta} r_{23} e^{i \beta}\right)^{2} t_{12} \\
& +t_{21} e^{i \beta} r_{23} e^{i \beta}\left(r_{22} e^{i \beta} r_{23} e^{i \beta}\right)^{3} t_{12}+\cdots \\
= & r_{11}+\frac{t_{12} t_{21} r_{23} e^{i 2 \beta}}{1-r_{22} r_{23} e^{i 2 \beta}},
\end{aligned}
$$

where $\beta=k d$ is the propagation phase, $d$ is the propagation distance from pattern layer to the ground plane, $d=h \cos \left(\arcsin \left(\sin \varphi / \sqrt{\varepsilon_{1} / \varepsilon_{2}}\right)\right), h$ is the vertical distance from pattern layer to the ground plane, $\varepsilon_{1}$ and $\varepsilon_{2}$ are the dielectric constants of areas 1 and 2, respectively, and $\alpha$ is the angle between the incident wave and the pattern layer. The electromagnetic wave to the bottom layer undergoes total 
reflection with a phase change of $180^{\circ}, r_{23}=-1$. Therefore, to achieve perfect absorption in the case of vertical and oblique incidence, the conditions to be met are

$$
\begin{aligned}
\left|r_{11}\right|-\left|t_{12} t_{21}-r_{11} r_{22}\right| & =0, \\
\theta\left|r_{11}\right|-\theta\left|t_{12} t_{21}-r_{11} r_{22}\right|-2 \beta=2 n \pi,|n| & =0,1,2, \ldots
\end{aligned}
$$

The scattering parameters obtained from the simulation are extracted and plotted as the curves shown in Figure 18. It can be seen that the amplitude difference is close to 0 for the full-band, and the phase difference is close to $0^{\circ}$ at the resonant frequency, so the phase and amplitude conditions are satisfied.

\section{Experimental Verification and Discussion}

5.1. Preparation of Resistive Film Absorber Sample. To verify the simulation results of the resistive film absorber, the ITO film was prepared by the magnetron sputtering deposition method, as shown in Figure 19. The substrate plate to be coated is the cathode, and the ITO target (anode) is below the dark chamber. There are orthogonal electric and magnetic fields that exist between the cathode and anode. The electric field causes the inert gas at the bottom right to divide into positive ions and electrons. The ions within the inert gas bombard the ITO target surface under the action of Lorentz force, and the sputtered atoms toward the substrate to form the film.

The ITO is patterned using laser etching, and the material properties of the glass make it more durable for laser ablation. Figure 20 shows the laser etching process with an accuracy of $0.05 \mathrm{~mm}$. The minimum gap of the proposed structure is $0.1 \mathrm{~mm}$, and the accuracy meets the demand.

5.2. Measurement and Discussion. We proceed to present experimental validations of the numerical results of section 3.3. The reflectivity of the sample was measured by the freespace method, as shown in Figure 21. To obtain plane wave excitation, the distance between the antennas and the sample should meet the far-field conditions as

$$
d>\frac{2 D^{2}}{\lambda},
$$

where $\lambda$ is the wavelength of the incident wave and $D$ is the length of the antenna diagonal.

At the same time, to eliminate as much as possible the effect of sample edge scattering on the reflection coefficient measurement accuracy, the size of the sample is $20 \mathrm{~cm} \times 20 \mathrm{~cm}$. The equipment required for the experiment are vector network analyzer and horn antennas; the range of measurement is limited by the upper frequency of vector network analyzer, so the maximum measurable frequency is $43 \mathrm{GHz}$. To eliminate reflection interference in the experimental environment, the reflection coefficient of a copper plate of the same size as the sample was first measured as a background value. Then, we maintained the same position, removed the copper plate, and measured the reflection coefficients of resistive film absorber again.

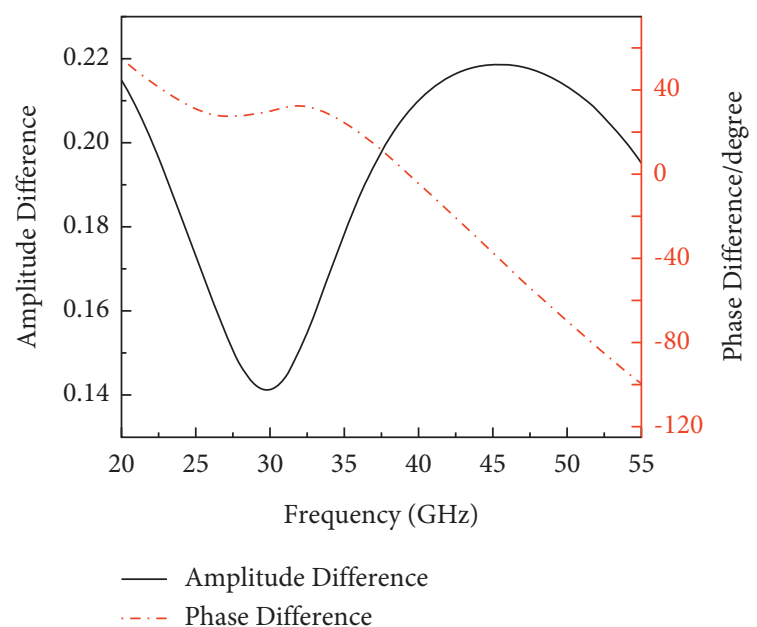

FIGURE 18: Amplitude (left column) and phase (right column) differences calculated by the multiple reflection model.

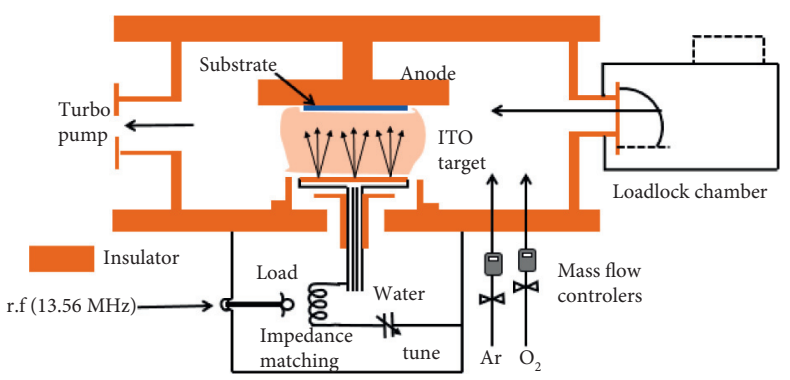

FIgURE 19: Schematic diagram of RF magnetron sputtering.

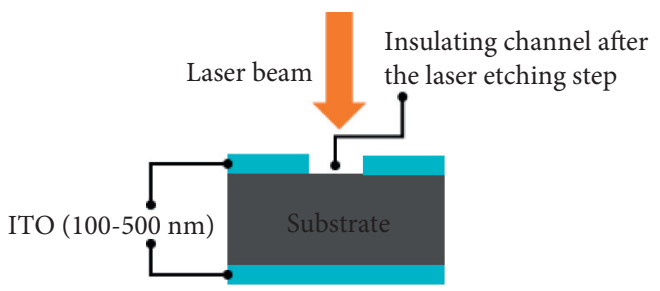

Figure 20: Laser etching process.

The orientations of both dual-ridge horn antennas were rotated by $0^{\circ}$ and $90^{\circ}$ to measure the reflection coefficient under TE and TM polarizations at vertical incidence. Figure 22(a) and 22(b) shows the results of $S_{11}$ measurement, respectively. The resonance points appear at $38 \mathrm{GHz}$ and 39.0 $\mathrm{GHz}$, respectively, and the resonant frequency and absorption bandwidth are similar to the simulation results. The absorption curves at different frequencies are shown in Figure 23, which proves the high absorption in both polarization modes. The experimental results differ from the simulation results due to the influence of the processed sample. High square resistance ITO is thin and the power of laser etching is difficult to adjust, which can easily lead to inadequate etching or damage the integrity of the structure edges.

The proposed resistive film absorber is compared with other miniaturized or broadband absorbers in Table 2, where 


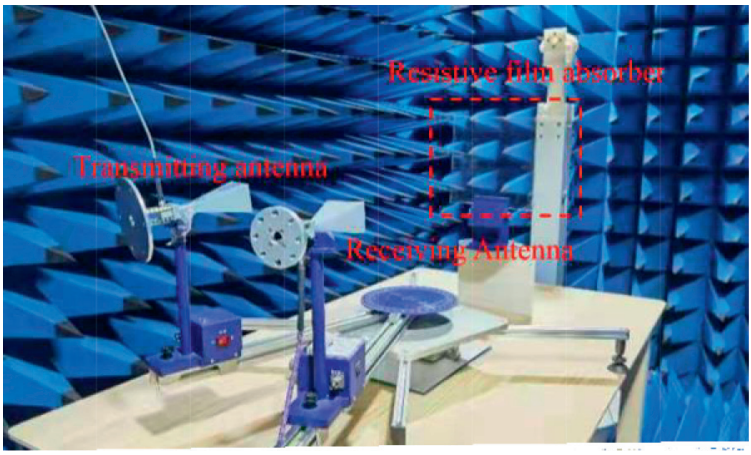

Figure 21: Photograph of the experiment setup.

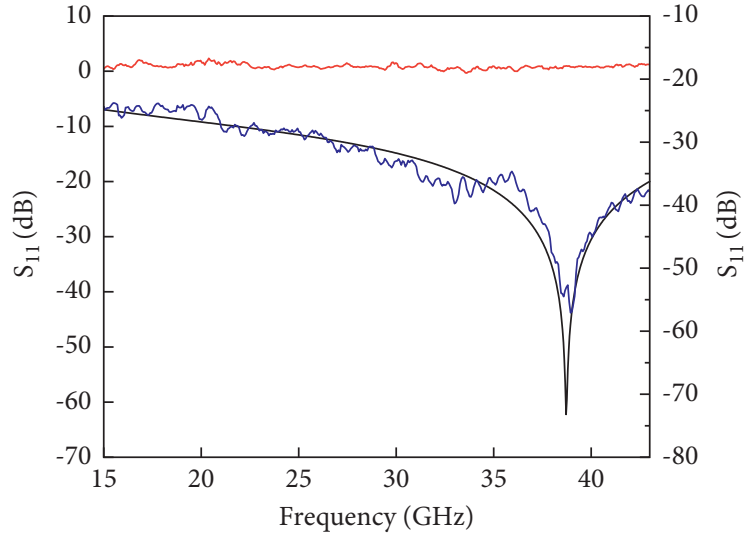

_ TE Polarization Simulation
_ TE Polarization Measurement

(a)

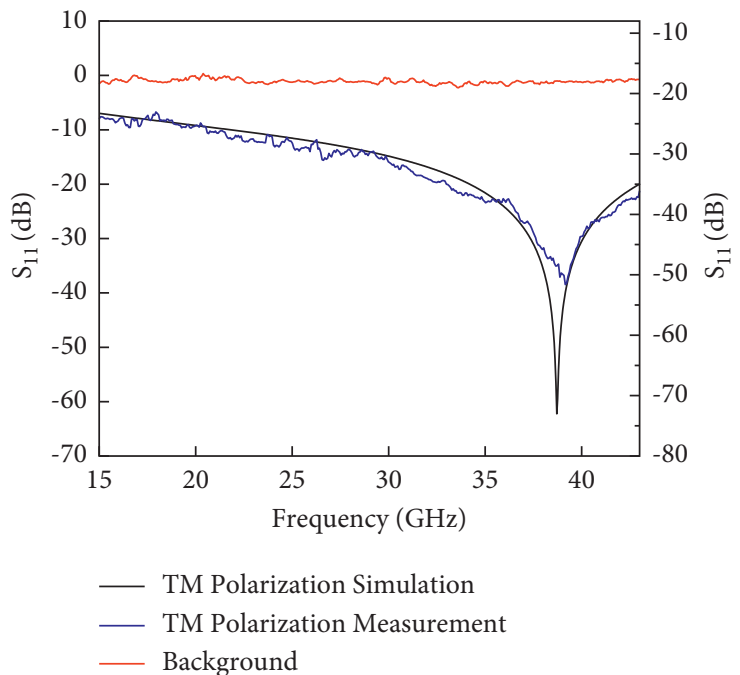

(b)

FIGURE 22: Simulation (left column) and measurement (right column) results: (a) TE polarization and (b) TM polarization.

the fractional bandwidth refers to the $-10 \mathrm{~dB}$ absorption bandwidth. The effective absorption bandwidth of traditional absorbers based on resonant of metal structures is narrow. The bandwidth can be extended by the multilayer structure, but it is not suitable for miniaturized scenarios. Broadband and miniaturized designs can be achieved by loading resistor elements, but processing is costly and complicated. Therefore, the proposed absorber has advantages in terms of broadband, miniaturization, and fabrication process.

\section{Application of Electromagnetic Interference Suppression}

To verify the radiation-suppression effect of the proposed structure, the absorber and flip chip model are cosimulated using CST Microwave Studio. The resistive film absorber structure is arranged periodically between PCB and heatsink, which broke the resonance cavity and absorbed the radiation generated by the PCB. As shown in Figure 24, we arrange the designed resistive film absorber in the form of a $16 \times 16$ array under the heatsink. The distance between the heatsink and the PCB is $1.6 \mathrm{~mm}$, so the absorber array is
$0.9 \mathrm{~mm}$ from the PCB. To maintain the thermal performance of the system, the absorber structure in the middle was removed to leave space for the thermal conductivity pad.

The suppression effect is verified by comparing the $3 \mathrm{~m}$ field radiation with and without loading the designed absorber. As shown in Figure 25, when the absorber is added at the bottom of the heatsink, the average attenuation of the radiation emission from $18 \mathrm{GHz}$ to $47 \mathrm{GHz}$ is $12.4 \mathrm{~dB}$, and the best radiation-suppression effect is about $18 \mathrm{~dB}$. Corresponding to the far-field results, Figure 26 shows the nearfield electric field distribution at $39 \mathrm{GHz}$. In contrast, the radiation generated by the cavity resonance between the heatsink and PCB is significantly mitigated.

Since the square resistance value of ITO can only be maintained in a specific range, Figure 27 illustrates the far-field results for different square resistance values. When the square resistance is 200 or $300 \Omega /$ sq, the farfield results increase in the high frequency, and the radiation-suppression effect decreases by $1.6 \mathrm{~dB}$ on average, but it is not affected at low frequency. The radiationsuppression effect of resistive film absorber is less affected by the square resistance of ITO, which means that the proposed absorber can be used to obtain significant 


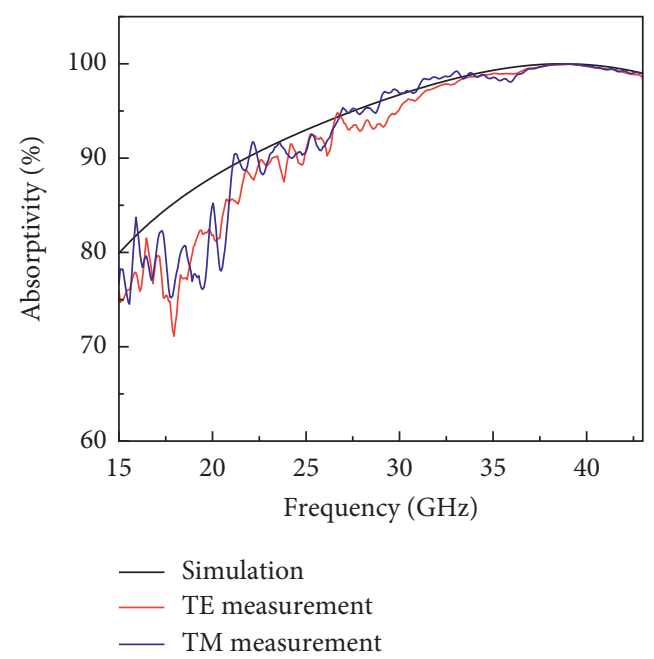

Figure 23: Absorptivity at different polarizations.

TABLE 2: Comparison with other metamaterial absorbers.

\begin{tabular}{|c|c|c|c|c|}
\hline & Techniques & Unit size & Thickness & Fractional bandwidth (\%) \\
\hline$[18]$ & Fractal structure & $0.151 \lambda_{\mathrm{L}}$ & $0.060 \lambda_{\mathrm{L}}$ & 23.0 \\
\hline [19] & Graphene & $0.110 \lambda_{\mathrm{L}}$ & $0.041 \lambda_{\mathrm{L}}$ & 20.3 \\
\hline$[20]$ & Multilayer structure & $0.286 \lambda_{\mathrm{L}}$ & $0.130 \lambda_{\mathrm{L}}$ & 61.3 \\
\hline [21] & Resistor-loaded & $0.063 \lambda_{\mathrm{L}}$ & $0.063 \lambda_{\mathrm{L}}$ & 105.6 \\
\hline$[22]$ & Asymmetric sectional resonator structures & $0.535 \lambda_{\mathrm{L}}$ & $0.054 \lambda_{\mathrm{L}}$ & 20.17 \\
\hline [23] & Resistive film & $0.128 \lambda_{\mathrm{L}}$ & $0.093 \lambda_{\mathrm{L}}$ & 118.8 \\
\hline$[24]$ & Multilayer structure and resistive film & $0.073 \lambda_{\mathrm{L}}$ & $0.082 \lambda_{\mathrm{L}}$ & 161.0 \\
\hline$[25]$ & Based on spoof surface plasmon polaritons & $0.355 \lambda_{\mathrm{L}}$ & $0.177 \lambda_{\mathrm{L}}$ & 63.7 \\
\hline This work & Resistive film & $0.140 \lambda_{\mathrm{L}}$ & $0.049 \lambda_{\mathrm{L}}$ & 89.5 \\
\hline
\end{tabular}
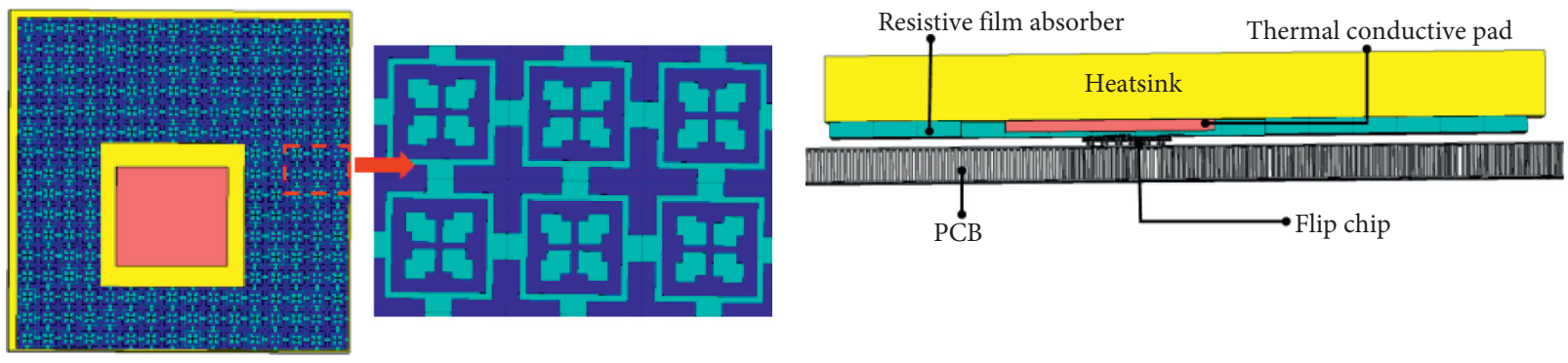

Heatsink

Thermal conductive pad

(a) (b)

FIGURE 24: (a) The arrangement of periodic structure of resistive film absorber on the heatsink and (b) cross-sectional view of heatsink with the proposed structure. 


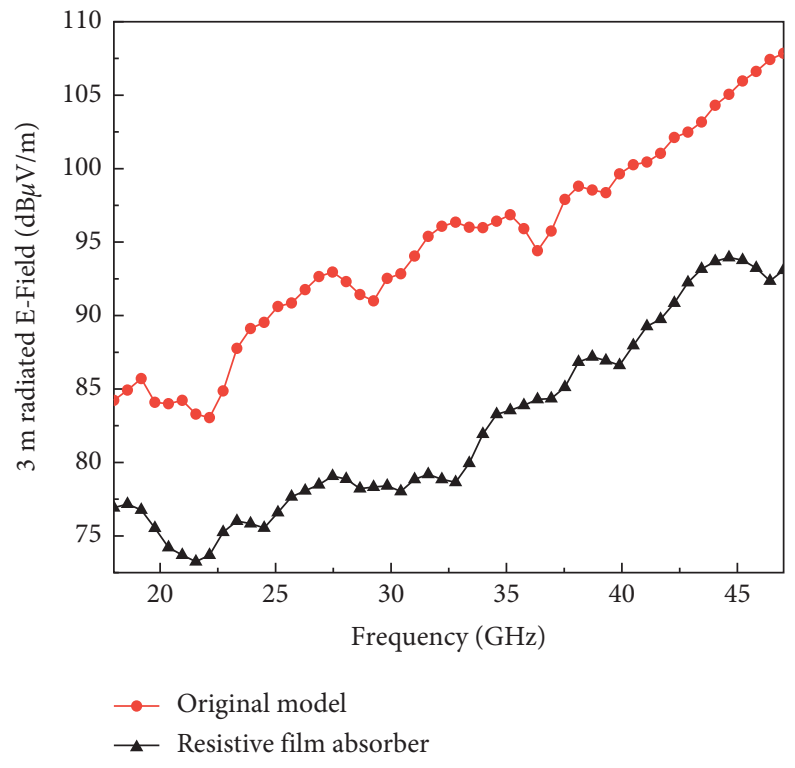

FIgURE 25: Radiated E-Field of package at $3 \mathrm{~m}$.

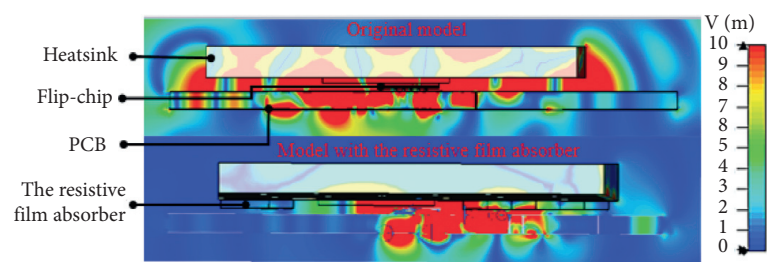

FIGURE 26: Near-field electric field distribution with and without resistive film absorber.

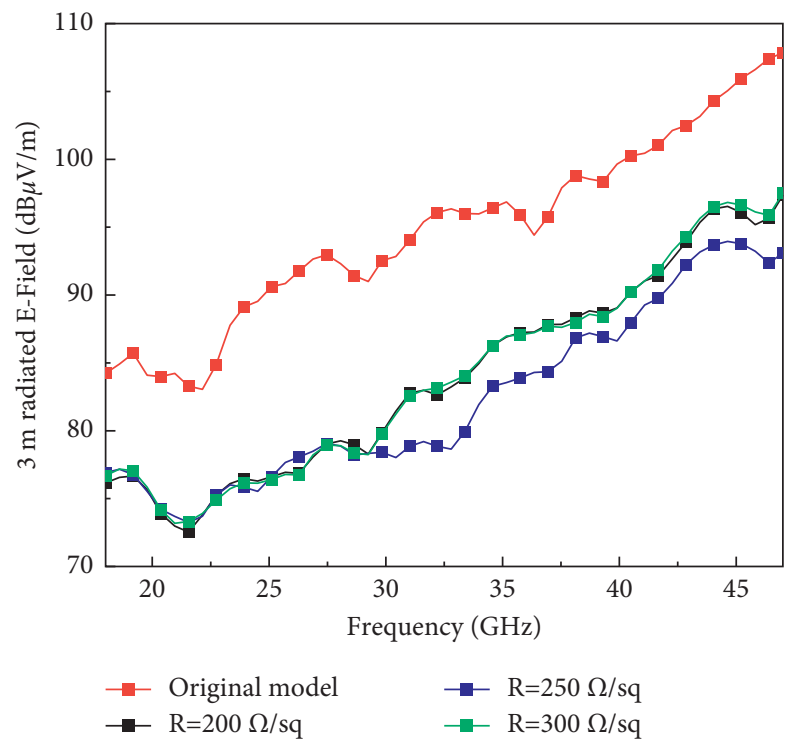

Figure 27: Radiated E-Field under different square resistance. 
radiation suppression in a high-speed chip packaging system.

\section{Conclusion}

In this paper, a miniaturized, polarization-insensitive resistive film absorber composed of Jerusalem crossshaped, ring-shaped, and L-shaped structures is proposed. The proposed structure achieves perfect absorption in broadband from $21 \mathrm{GHz}$ to $55 \mathrm{GHz}$. We analyze the absorption mechanism in terms of both impedance matching and the loss of incident wave. The measurement results verify the accuracy of theoretical derivation and full-wave simulation, and the cosimulation results verify the effective electromagnetic interference absorption and suppression capability of the proposed resistive film absorber, and the undesired radiation is suppressed by $18 \mathrm{~dB}$, showing that the proposed absorber can apply to the radiation suppression of future miniaturized electronic devices.

\section{Data Availability}

The data used to support the findings of this study were supplied by Shuyun Huo under license and so cannot be made freely available. Requests for access to these data should be made to (Shuyun Huo, 201931903030@ stu.hebut.edu.cn).

\section{Conflicts of Interest}

The authors declare that they have no conflicts of interest.

\section{Acknowledgments}

This work was supported by the Natural Science Foundation of China (grant no. 62071424), Zhejiang Provincial Natural Science Foundation of China (ZPNSFC) (grant no. LD21F010002), and Natural Science Foundation of China (grant no. 62027805).

\section{References}

[1] A. Genc, H. Basyigit, I. B. Basyigit, and S. Helhel, "Heatsink preselection chart to minimize radiated emission in broadband on the PCB," IEEE Transactions on Electromagnetic Compatibility, vol. 63, no. 2, pp. 419-426, 2021.

[2] X. He and T. H. Hubing, "A closed-form expression for estimating the maximum radiated emissions from a heatsink on a printed circuit board," IEEE Transactions on Electromagnetic Compatibility, vol. 54, no. 1, pp. 205-211, 2012.

[3] Y. Li, W. Cao, P. Zuo et al., "Analysis of multilayer structure near- and far-field radiation by the coupled PP-peec and fieldequivalence principle method," IEEE Transactions on Electromagnetic Compatibility, vol. 61, no. 2, pp. 495-503, 2019.

[4] P. Cheng, E.-P. Li, L. Zhang et al., "Electromagnetic and thermal characteristics of graphite for radiation suppression in wire-bonded package heat sink," IEEE Transactions on Electromagnetic Compatibility, vol. 60, no. 5, pp. 1445-1453, 2018.
[5] Y.-J. Chou, L.-S. Chen, and M.-P. Houng, "High-frequency noise absorption of Ag-Fe3O4 films on microstrip transmission line," IEEE Transactions on Magnetics, vol. 51, no. 4, pp. 1-4, 2015.

[6] Y. Wu, M. Han, M. Liu, and L. Deng, "Large electromagnetic wave absorbing bandwidth of composites containing Fe3O4 nanoribbons," IEEE Transactions on Magnetics, vol. 51, no. 11, pp. 1-4, 2015.

[7] M. Kim, "A compact EBG structure with wideband power/ ground noise suppression using meander-perforated plane," IEEE Transactions on Electromagnetic Compatibility, vol. 57, no. 3, pp. 595-598, 2015.

[8] L.-F. Shi, K.-J. Li, H.-Q. Hu, and S. Chen, "Novel L-EBG embedded structure for the suppression of SSN," IEEE Transactions on Electromagnetic Compatibility, vol. 58, no. 1, pp. 241-248, 2016.

[9] P. Zuo, T. Li, M. Wang, H. Zheng, and E.-P. Li, "Miniaturized polarization insensitive metamaterial absorber applied on EMI suppression,” IEEE Access, vol. 8, pp. 6583-6590, 2020.

[10] Y. Cheng, F. Chen, and H. Luo, "Triple-band perfect light absorber based on hybrid metasurface for sensing application," Nanoscale Research Letters, vol. 15, no. 1, p. 103, 2020.

[11] H. Zhang, Y. Cheng, and F. Chen, "Quad-band plasmonic perfect absorber using all-metal nanostructure metasurface for refractive index sensing," Optik, vol. 229, Article ID 166300, 2021.

[12] Y. C. Hui, C. Q. Wang, and X. Z. Huang, "Design and fabrication of broadband radar metamaterial absorber based on the resistor FSS," Acta Physica Sinica, vol. 64, no. 21, Article ID 218102, 2015.

[13] J. B. O. de Araújo, G. L. Siqueira, E. Kemptner, M. Weber, C. Junqueira, and M. M. Mosso, "An ultrathin and ultrawideband metamaterial absorber and an equivalent-circuit parameter retrieval method," IEEE Transactions on Antennas and Propagation, vol. 68, no. 5, pp. 3739-3746, 2020.

[14] J. Chen, Z. Hu, G. Wang et al., "High-impedance surfacebased broadband Absorbers with interference theory," IEEE Transactions on Antennas and Propagation, vol. 63, no. 10, pp. 4367-4374, 2015.

[15] D. R. Smith, D. C. Vier, T. Koschny, and C. M. Soukoulis, "Electromagnetic parameter retrieval from inhomogeneous metamaterials," Physical review. E, Statistical, nonlinear, and soft matter physics, vol. 71, no. 3, Article ID 036617, 2005.

[16] Z. Zhou, K. Chen, B. Zhu, J. Zhao, Y. Feng, and Y. Li, "Ultrawideband microwave absorption by design and optimization of metasurface salisbury screen," IEEE Access, vol. 6, pp. 26843-26853, 2018.

[17] F. Costa, S. Genovesi, A. Monorchio, and G. Manara, "A circuit-based model for the interpretation of perfect metamaterial absorbers," IEEE Transactions on Antennas and Propagation, vol. 61, no. 3, pp. 1201-1209, 2013.

[18] G. Shuai, B. Su, and X. Zhao, "Planar isotropic broadband metamaterial absorber," Journal of Applied Physics, vol. 114, no. 16, Article ID 163702, 2013.

[19] Y. Zhang, Y. Li, Y. Cao, Y. Liu, and H. Zhang, "Graphene induced tunable and polarization-insensitive broadband metamaterial absorber," Optics Communications, vol. 382, pp. 281-287, 2017.

[20] D. Fei, Y. Cui, X. Ge, Y. Jin, and S. He, "Ultra-broadband microwave metamaterial absorber," Optics Communications, vol. 100, no. 10, Article ID 103506, 2012.

[21] D. Kundu, A. Mohan, and A. Chakrabarty, "Single-layer wideband microwave absorber using array of crossed 
dipoles," IEEE Antennas and Wireless Propagation Letters, vol. 15, pp. 1589-1592, 2016.

[22] Q. Wang and Y. Cheng, "Compact and low-frequency broadband microwave metamaterial absorber based on meander wire structure loaded resistors," AEU -International Journal of Electronics and Communications, vol. 120, Article ID 153198, 2020.

[23] Y. Z. Cheng, H. Luo, and F. Chen, "Broadband metamaterial microwave absorber based on asymmetric sectional resonator structures," Journal of Applied Physics, vol. 127, no. 21, Article ID 214902, 2020.

[24] Y. Tayde, M. Saikia, K. V. Srivastava, and S. A. Ramakrishna, "Polarization-insensitive broadband multilayered absorber using screen printed patterns of resistive ink," IEEE Antennas and Wireless Propagation Letters, vol. 17, no. 12, pp. 2489-2493, 2018.

[25] Y. Pang, J. Wang, H. Ma et al., "Spatial k-dispersion engineering of spoof surface plasmon polaritons for customized absorption," Scientific Reports, vol. 6, no. 1, Article ID 29429, 2016. 